\title{
Analytical and Numerical Investigation of Hardening Behavior of Porous Media
}

\author{
Younis Khalid Khdir* \\ Department of Mechanical and Energy, Erbil Technology Engineering College, Erbil Polytechnic University, Kurdistan Region, Iraq
}

\author{
${ }^{*}$ Corresponding author: \\ Younis Khalid, Department \\ of Mechanical and Energy, \\ Erbil Technology Engineering \\ College, Erbil Polytechnic \\ University, Kurdistan Region, \\ Iraq. 07504790685. \\ E-mail: younis.khdir@epu. \\ edu.iq \\ Received: 18 April 2019 \\ Accepted: 16 July 2019 \\ Published: 30 October 2019 \\ DOI \\ 10.25156/ptj.v9n2y2019.pp1-10
}

\section{A B S TR A C T}

In this study, a comparative analysis is presented between a new proposed analytical model and numerical results for macroscopic behavior of porous media with isotropic hardening in its matrix. The macroscopic behavior of a sufficiently large representative volume element, with 200 identical spherical voids, was simulated numerically using finite element method and compared with elementary volume element that contains one void. The matrix of the porous material is considered as elastoplastic with isotropic hardening obeys exponential law for isotropic hardening. A New Parameter $B$ was added with exponential law for isotropic hardening to represent the new proposed analytical model for macroscopic isotropic porous hardening. The new added parameter $B$ depended only on the porosity. The results of the new proposed analytical model were compared with numerical results for different types of cyclic loading. Very good agreements were found between the numerical results and the proposed analytical model.

Keywords: Elastic-plastic porous materials; Computational homogenization; Non-linear isotropic hardening; Exponential law; Three-scale homogenization.

\section{INTRODUCTION}

Most of the engineering components and structures are subjected to cyclic loadings. It is important to take cyclic plasticity and hardenings into account, so as to be able to predict the fatigue life of components under the cycle fatigue regime, (Ristinmaa, 1995, Sorić et al., 2000, Chaboche et al., 2012).

Other authors were studied the mechanical behavior of composite materials under cyclic loading. Samrout presented a model for a steel composite under cyclic loading (Samrout et al., 1997), Zhao studied aluminum alloy with considering hardening, they tried to simulate spring back using a combined kinematic/isotropic hardening model. The material parameters in the hardening model were identified by an inverse method using a three-point bending test. The test was conducted on aluminum sheet $\mathrm{Al}$ 6022-T4 (Zhao and Lee, 2001). Doghri studied two-phase elastoplastic materials under cyclic loading (Doghri and Ouaar, 2003, Doghri and Friebel, 2005). Zhang and Verma presented cyclic plasticity model for mixed hardening where they considered a small strain micromorphic elastoplastic model with isotropic/kinematic hardening for modeling the size effect and Bauschinger effect in material with microstructure. They used two numerical examples, included a thin film and a plate with underlying structures subjected to cyclic loading. They were analyzed to verify the theoretical developments and numerical formulations. They studied plastic behaviors in micromorphic continuum, such as Bauschinger effect, size effect, and ratcheting effect (Zhang et al., 2011, Verma et al., 2011). Several authors also studied the kinematics hardening properties, (Chaboche and Jung, 1997, Besson and Guillemer-Neel, 2003, Besson, 2010, Rezaiee-Pajand and Sinaie, 2009, Mosler, 2010, Mahmoudi et al., 2011, Hashemi and Farshi, 2011, De Angelis, 2012).

In 2013, Balan and Cazacu considered strain rate for porous materials in the matrix phase. They noted that a very good description of the plastic behavior for large strains can be obtained using strain-rate potentials, and such formulations are particularly suitable for design optimization.

A thermodynamically consistent multi-scale, ratedependent, and non-local approach was developed by Voyiadjis and Song, 2002. A simple analytical model of void growth was introduced for studying the behavior of a single cylindrical or spherical-ellipsoidal void in a plastic material and also of any three-dimensional (3D) void in a porous material containing a periodic array of voids. They presented a simple analytical model of void growth for studying the behavior of a single spherical/ellipsoidal void in a plastic material. They used a $3 \mathrm{D}$ void in a porous material containing a periodic array of voids. The effect of the stress triaxiality on the void growth has been taken 
into account in the model together with the macro- and microscopic factors such as the hardening exponent, the stress components, the volume fraction of voids, the void shape and spacing, and the constitutive softening (Dung, 1992).

There is a lot of studies about porous materials. Some of them deal with plasticity models. The classical plasticity model of Gurson model contains the porosity as internal variable and the author considered perfect plastic in the matrix of porous materials (Gurson, 1977). Tvergaard proposed the extended yield criteria of Gurson type model in two different studies (Tvergaard and Needleman, 1984, Tvergaard, 1982), so called Gurson-Tvergaard-Needleman (GTN) model, with the new parameters $q$, with $i=1$, 2,3 , to adjust their experiments, and, it has been used most frequently. In the GTN model, isotropic hardening is accounted for the flow stress of the matrix material $\sigma_{\mathrm{Y}}$, while in Tvergaard and Needleman, 1984, study, the modification of $f^{*}$ (void coalescence) was proposed as a piecewise linear function of the initial porosity $f$. The value of $f^{*}$ showed an increase of porosity due to the coalescence of the voids, and then the critical void volume fraction $f_{c}$ has been extended. The non-uniform transformation field analysis was used and the non-uniform distribution of local plastic strain in the solid matrix was taken into account by Jiang et al., 2011. A new pressure-dependent yield function was proposed by introducing a plastic Poisson's ratio within the theoretical formulation of the plastic potential to derive the equivalent stress-strain curve and an exponential hardening law was introduced. The multi-axial problem was reduced to a uniaxial equivalent one, while, the parameters reduced to two. They presented that the experimental curves can be used for parameters identification (Carollo et al., 2016).

Studying hardening properties for porous materials also are one of the interesting subjects by other authors. Becker and Needleman (1986) and Mear and Hutchinson (1985) proposed the linear hardening into Gurson model (Becker and Needleman, 1986, Mear and Hutchinson, 1985). Ristinmaa presented the comparison between Gurson model and unit cell model (Ristinmaa, 1997), while Becker and Needleman, 1986, indicated the void growth during isotropic and kinematic hardening for porous materials they used the same principal of Ristinmaa. Another analytical function, for spherical void in a spherical volume element, was proposed by Leblond, the author considered incompressible isotropic and kinematic hardening matrix material (Leblond et al., 1995). They were proposed an extension of GTN model. Later, the same principle is used by Jin et al., 2008, Seifert and Schmidt, 2008, Seifert and Schmidt, 2009, Siad et al., 2009. They studied the evaluation of the yield surface of porous materials, using elementary volume element containing one void, using finite element method. A 3D unit cell, with one void, is simulated by Kuna and Sun, 1996, considering void growth in ductile materials. Steglich compared calculation of a unit cell with Gurson and porous metal plasticity model (LPD) with examining hardening, using continuum damage mechanics (Steglich et al., 2005). Chawla discussed the general effects of porosity on the mechanical behavior (tensile and fatigue) of a specific composite. The discussed studies focus on the calculation of a unit cell and evaluate the damage under cyclic loading with hardening and some other cases and they discussed the loading stories (Chawla and Deng, 2005). They found that increasing sintered density resulted in lower pore fraction, more spherical pore shape, and smaller average pore size. They noticed that increasing pore size was correlated directly with an increase in the irregularity of pore shape.

Isotropic hardening model for matrix phase used in this study is isotropic hardening as in Equation 1 and, for non-linear hardening, the most common equations used for isotropic hardening are power law (Ludwik law), Equation 2, and the exponential law (Voce law), Equation 3, which were equations used recently by several authors, for example, Chaboche, 1986, Simo and Hughes, 2006, Steglich et al., 2005, Allain and Bouaziz, 2008, Seifert and Schmidt, 2008, Seifert and Schmidt, 2009, Cardoso and Yoon, 2009, Taherizadeh et al., 2009, Cao et al., 2009, Leu and Li, 2012, Chaaba, 2013. Linear and non-linear evolutions of isotropic hardening, for the matrix phase only, can be calculated according to the equations below, power law or exponential law, respectively:

$$
\begin{aligned}
& \sigma_{\mathrm{Y}}=\sigma_{\mathrm{o}}+\mathrm{H} \varepsilon^{\mathrm{p}} \\
& \sigma_{\mathrm{Y}}=\sigma_{\mathrm{o}}+\mathrm{H}\left(\varepsilon^{\mathrm{p}}\right)^{\mathrm{b}} \\
& \sigma_{\mathrm{Y}}=\sigma_{\mathrm{o}}+\mathrm{Q}\left(1-\mathrm{e}^{-\mathrm{b} \varepsilon^{\mathrm{p}}}\right)
\end{aligned}
$$

For porous materials, in this paper, it is considered that the matrix phase obeys Voce law of isotropic hardening, where $\sigma_{Y}$ is the macroscopic uni-axial stress, and $Q$ corresponds to the amplitude of the exponential function (saturation hardening, positive or negative). $b$ denoted the coefficient of decay (rate of saturation hardening). $H$ is material parameter (plastic hardening modulus), e $e^{\phi}$ represents the microscopic equivalent plastic strain, and $\sigma_{0}$ the initial yield stress of the pure matrix. $Q$ and $b$ can be adjusted to a given material. The sum of $Q$ and $\sigma_{0}$ is denoted as the maximal yield surface radius in deviatory stress space. $b$ governs the shape of the hardening curve between the lower $\sigma_{0}$ and the upper $\left(\sigma_{0+\varrho}\right)$ bounds. Increasing the value of $b$ leads to reach the upper bound faster, for $Q=0$ or $b=0$, isotropic 
hardening does not occur. The variable $p$ is the cumulated plastic strain. Isotropic hardening is applied to the yield as follows:

$$
\sigma_{\text {iso }}=\sigma_{\text {eq }}-\sigma_{\mathrm{Y}}
$$

$\sigma_{e q}$ has the same scalar measure of the von Mises stress equivalent, Equation 6.

Assume, at this point, that the material used is a plastic porous with porosity $\mathrm{f}$. The deviatory von Mises equivalent stress $\sum_{\text {eq }}$ and the hydrostatic part of the macroscopic stress tensor $\sum_{\text {eq }}$ are:

$$
\begin{aligned}
& \Sigma_{\mathrm{m}}=\frac{1}{3} \operatorname{tr}(\bar{\sigma})=\frac{\sigma_{11}+\sigma_{22}+\sigma_{33}}{3} \\
& \Sigma_{\text {eq }}=\sqrt{\frac{\left.\left(\sigma_{11}-\sigma_{22}\right)^{2}+\left(\sigma_{11}-\sigma_{33}\right)^{2}+\sigma_{33}\right)^{2}}{2}}
\end{aligned}
$$

The equivalent plastic strain $\varepsilon^{b}$ can be determined according to the equation below, (Buryachenko, 1996, Sorić et al., 2000, Besson and Guillemer-Neel, 2003, Steglich et al., 2005, Chaboche, 2008, Jin et al., 2008, Seifert and Schmidt, 2008, Seifert and Schmidt, 2009):

$$
\begin{aligned}
& \varepsilon^{\mathrm{p}}=\int_{t=0}^{\mathrm{t}} \dot{\varepsilon}^{\mathrm{p}} \mathrm{dt} \\
& \dot{\varepsilon}^{\mathrm{p}}=\sqrt{\frac{2}{3} \dot{\varepsilon}_{\mathrm{ij}}^{\mathrm{p}} \dot{\varepsilon}_{\mathrm{ij}}^{\mathrm{p}}}
\end{aligned}
$$

where $t$ represents time and is plastic strain rate.

Several authors used the same principle of Voce law for estimating hardening properties and they proposed the material parameters $(Q, b)$ for different materials and composites, for example, Chow and Yang, 2003, Allain and Bouaziz, 2008, Cardoso and Yoon, 2009, Taherizadeh et al., 2009, Cao et al., 2009, Rousselier et al., 2010, Leu and Li, 2012, Chaaba, 2013. Based on Equation 3, the relationship between the equivalent stress and the equivalent strain or multi-component strain hardening model can be expressed as:

$$
\Sigma_{\text {eq }}=\sigma_{o}+\sum_{\mathrm{i}} \mathrm{Q}_{\mathrm{i}}\left(1-\mathrm{e}^{-\mathrm{b}_{\mathrm{i}} \varepsilon^{\mathrm{p}}}\right)
$$

For example, Lademo et al., 1999, Chow and Yang, 2003.

The objective of this study is to propose an analytical equation that is able to describe macroscopic hardening properties for porous materials under tension or compression or tension-compression. In this study, a new general analytical model is proposed which can be used for porous materials with different porosities and volume fraction through using numerical calculations finite element for a $3 \mathrm{D}$ representative volume element (RVE).

The results in this study obtained through performing numerical finite element results of a sufficiently large 3D RVE of porous material. The considered volume contains 200 randomly distributed identical spherical voids. An elementary volume element, with one centered spherical void, is also considered in the finite element simulation. The proposed analytical model is an extension of the exponential law for isotropic hardening of the pure matrix. This macroscopic model is proposed based on the numerical calculations of 3D RVE finite element method. A new parameter is added to the exponential law for hardening in the matrix model, to adjust the analytical results from exponential law to the obtained results from numerical finite element. The numerical results are obtained in the case of simple tension tests for different porosities considering isotropic hardening in the matrix phase. Then, to test the efficiency of the proposed macroscopic analytical model, it compared in the case of cyclic loading (tension and compression) with numerical results. The equivalent stress $\Sigma_{e q}$, as explained in equation 9, is described using the multi-component strain hardening model. The yield stress and strain hardening constants are fitted to the uniaxial stress-strain curves in a reference.

The motivation of proposing this analytical model is there are few studies about isotropic of porous materials, which is an important subject. However, there are a lot of studies conducted about isotropic hardening for materials and composites as well, for example, Khan and Jackson, 1999, Verleene et al., 2002, Khoei and Azami, 2005, Berisha et al., 2010, Kossa and Szabó, 2009. Hence, in this paper, an analytical model for isotropic porous hardening is proposed.

The present paper is organized as follows; in section 2, the investigated microstructure, computational method, and proposed analytical model are presented. The results are presented and discussed in section 3; some concluding remarks are given in section 4 .

\section{COMPUTATIONAL HOMOGENIZATION}

The procedure of creating 3D RVE with FE mesh for porous materials is presented here. Indicating materials properties to the matrix are one of the essential steps toward presenting the modeling and numerical analysis. The procedure for defining boundary conditions is explained. The mesh size and the steps of the calculation for different 
microstructures and different volume fractions are also explained in detail in this section.

\section{Microstructure and Mechanical Behavior}

Materials considered in this study are made of a plastic matrix containing identical spherical voids randomly distributed. The microstructure is plastic matrix with random or periodically distribution of voids, through studying RVE, containing 200 randomly distributed voids or elementary volume elements contains one centered void, respectively, Figure 1. The Young's modulus of the matrix $E=207 \mathrm{MPa}$ and the Poison ratio $v=0.3$ are assumed. The saturation hardening $Q=200$ and the rate of saturation hardening $b=10$, which supposed to fulfill the von Mises plasticity model with initial yield stress $\sigma_{0}=245 \mathrm{MPa}$ is performed, considering that there is isotropic hardening, and there is no overlapping between the voids, while the random distribution of 200 voids in an RVE represents random microstructure and elementary volume element which contains only one void represents a periodic microstructure.

\section{Creating Microstructure}

Several schemes will be possible to generate microstructures with matrix and spherical voids, distributed periodically or randomly, which can be defined by the radius and coordinates of the centers of the spherical voids. The FE method was chosen for the numerical computations. The FE calculations were carried out with Zebulon FE software. The obtained microstructure consists of randomly or periodic distributed non-overlapping identical spherical voids embedded in the matrix. In Figure 1, a cubic microstructure deals about elementary volume element with one centered void and the large RVE size containing 200 voids with volume fractions $f=0.23$.

\section{Boundary Conditions}

The second important issue for the numerical tests, after generating microstructures, concerns the boundary conditions. For a uni-axial tensile loading in the $\mathrm{x}$ direction, for example, Figure 2 prescribed these conditions as follows:

$$
\begin{aligned}
& \mathrm{u}\left\{\begin{array}{l}
\text { pla n e } \\
(\mathrm{x}=0, \mathrm{y}, \mathrm{z})
\end{array}\right\}=0 \mathrm{v}\left\{\begin{array}{l}
\text { p o in } \mathrm{O} \\
(0,0,0)
\end{array}\right\}=0 \\
& \mathrm{u}\left\{\begin{array}{l}
\mathrm{pla} \mathrm{n} \mathrm{e} \\
(\mathrm{x}=1, \mathrm{y}, \mathrm{z})
\end{array}\right\}=\delta \mathrm{w}\left\{\begin{array}{l}
\text { p oin t } \\
\text { O }(0,0,0)
\end{array}\right\}=0 \\
& \mathrm{v}\left\{\begin{array}{l}
\text { p oin t } \\
\mathrm{A}(0,0,1)
\end{array}\right\}=0 \mathrm{w}\left\{\begin{array}{l}
\text { p oin t } \\
\mathrm{B}(0,1,0)
\end{array}\right\}=0
\end{aligned}
$$

in which, $\mathrm{u}, \mathrm{v}$ and $\mathrm{w}$ are the applied displacements in the, $\mathrm{x}, \mathrm{y}$, and $\mathrm{z}$ directions, $l$ is the RVE length, and $\delta$ is the prescribed displacement.
Then, in the tri-axial case, the boundary conditions have been controlled triaxiality. Overall triaxiality varied through changing two parameters, $\alpha$ and $\beta$. For the simulation time tof the boundary conditions, the following relations have been used. For more detail about choosing typical boundary condition, see (Besson et al., 2006, Fritzen et al., 2012, Khdir et al., 2013, Khdir et al., 2014, Khdir et al., 2015):

$$
\begin{aligned}
& \varepsilon_{11}(\mathrm{t})=\mathrm{t} \dot{\varepsilon}_{0}(\alpha+\beta) \\
& \varepsilon_{22}(\mathrm{t})=\mathrm{t} \dot{\varepsilon}_{0}(-\alpha+\beta) \\
& \varepsilon_{33}(\mathrm{t})=\mathrm{t} \dot{\varepsilon}_{0} \beta \\
& \sigma_{12}(\mathrm{t})=\sigma_{13}(\mathrm{t})=\sigma_{23}(\mathrm{t})=0.0
\end{aligned}
$$

Here, $\varepsilon_{0}>0$ is a prescribed deformation rate.

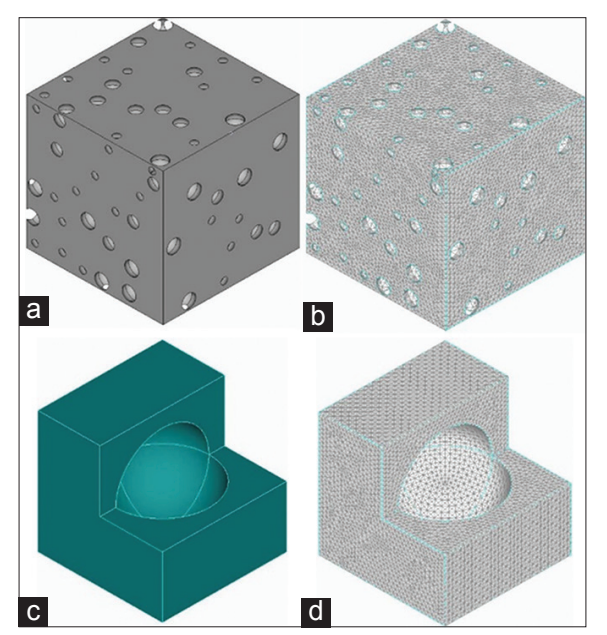

Figure 1: Examples of porous microstructures contain porosity of $f=0.23$, with mesh and without mesh, ( $a$ and $b)$ representative volume element with 200 random voids and (c and d) cross-section of elementary volume with one centered void

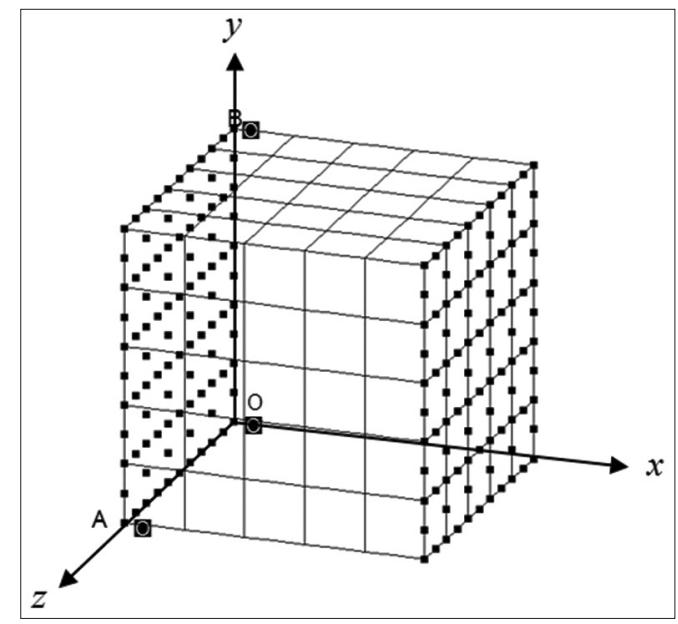

Figure 2: Description of boundary conditions 
The shear components considered of the macroscopic stress tensor are equal to zero. The hydrostatic stress and von Mises stress depend only on the diagonal components of the macroscopic stress.

\section{RESULTS AND DISCUSSION}

\section{Behavior Law of Pure Matrix}

For non-porous plastic material, pure matrix, with isotropic hardening, the user behavior law is shown in Figure 3, in the case of cyclic loading. This exponential law is given by Equation 3. This behavior law is used for the matrix during our studied porous materials. Noting that, for each cycle, after hardening, the accumulated stress is employed for calculating the new yield stress $\sigma_{0}$.

\section{The Macroscopic Behavior of Porous Materials}

In this section, the macroscopic behavior of porous materials is presented, using numerical calculations for 3D RVE. A new parameter $B$ is proposed and calculated to construct an analytical macroscopic behavior model for the studied porous material. Our new proposed model is, simply, the exponential law, of pure matrix, multiplying by the new determined parameter $B$, as shown in the following equation:

$$
\sigma_{\mathrm{Y}}=\mathrm{B}\left(\sigma_{\mathrm{o}}+\mathrm{Q}\left[1-\mathrm{e}^{-\mathrm{b} \varepsilon^{\mathrm{p}}}\right]\right)
$$

Based on the same strain methods, the equation evaluated, which explains the relationship between the porosity $f$ and the new proposed parameter $B$. Depending on the numerical results; the analytical model of exponential law is adjusted, Equation 10. To verify the efficiency of the new proposed parameter $B$, the numerical and the analytical results, using Equation 12, are plotted in Figure 4, for different porosities: $f^{\prime} 0.05, f=0.13$, and $f=0.23$. This figure indicates that the proposed analytical model can be used to predict the macroscopic stress-strain curves for porous materials with isotropic hardening.

The new proposed parameter $B$ can be determined according to the following equation:

$$
\mathrm{B}=\mathrm{q}_{1}(1-\mathrm{f})-\mathrm{q}_{2}
$$

Then, for calculating the exact values of the new proposed parameter $B$, in each case, some points were taken for the same plastic strain for all the tested RVE with different porosities, as indicated if Figure 5a. According to numerical tests, the values of were evaluated for each stage of isotropic hardening and for different porosities: $f=0, f=0.5$, $f=0.13$, and $f=0.23$. The relationship between the new calculated parameter $B$ and the used porosities has been plotted in Figure 5b. Then found that the new results are

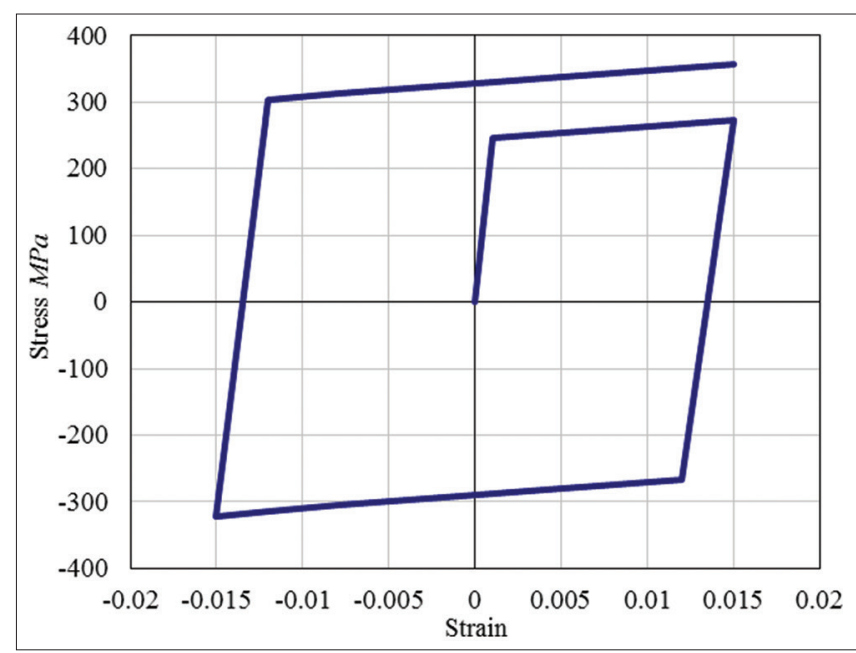

Figure 3: Behavior law of pure matrix, cyclic loading

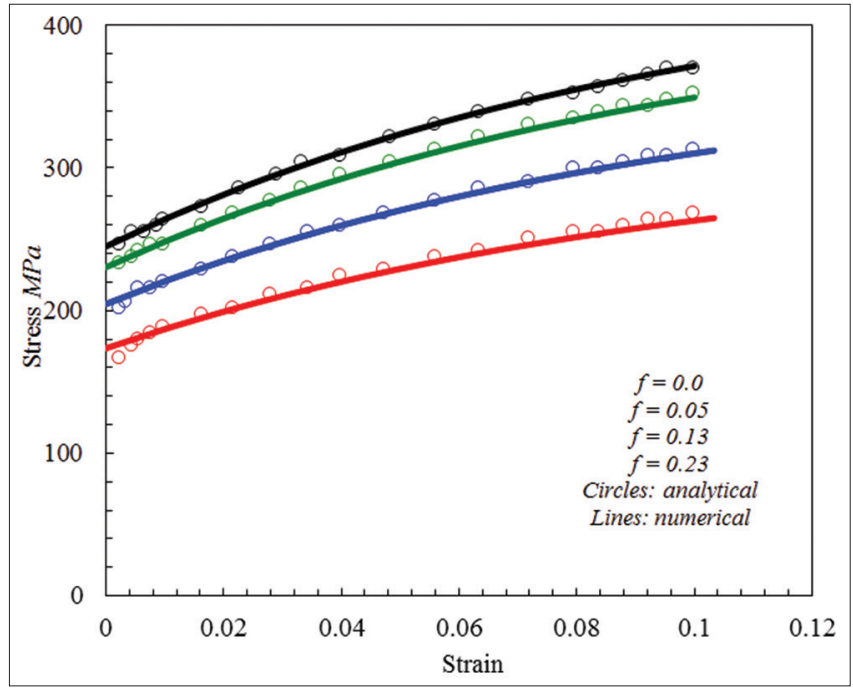

Figure 4: Comparison between analytical and numerical results for porous material with isotropic hardening

almost the same in each case. The average of parameters, in each stage of same plastic strain, is used to evaluate the relationship between the porosities and the values of the new proposed parameter $B$. Illustrating that, this parameter is strongly depend on the porosity. Depend on using numerical method, a mathematical equation is found, so as to correctly predict the values of the new proposed parameter $B$. The values of $q_{1}$ and $q_{2}$ are determined as: $q_{2}=1.263$ and $q_{2}=0.26$. The error percent is $<0.006 \%$.

\section{Comparison between Numerical FE Results and Analytical Model with Isotropic Hardening under Cyclic Loading}

Three different volume fractions were tested numerically using 3D RVE to predict the non-linear isotropic hardening and under cyclic loading, then compared with analytical results using the same proposed parameter $B$ with exponential law; details about the comparison are 
presented and very good matching between the numerical and analytical results is shown in Figure 6.

In Figure 6a, the tension-compression stress-strain resulted in three different volume fractions of porous materials with perfect plastic without pores is presented. It has been noticed that the yield stress decreases according to the increase of porosity, because of the structure change, while the relation remains parallel between the porous materials and plastic material without pores. Then, the same relations were plotted, as shown in Figure 3, while the results (stress-strain curves) obtained based on the analytical model (Equation 8) using the values of the new proposed parameter $B$, as shown in Figures 6, the analytical results are almost resemble the numerical finite element results of 3D RVE. To show clearly the comparison between the new analytical model and numerical finite element results, the results of each volume fraction are plotted separately. The results of plastic matrix, the three different volume fractions $f=0.5, f=0.13$, and $f=0.23$ are plotted in Figure 6, respectively. Then, the efficiently of the new model with the new proposed parameter $B$ with different volume fractions is interested. The proposed analytical model for porous materials can be used for different volume fractions, which is between 0.05 and 0.23 .

\section{Comparison between Numerical Result and Analytical Model for RVE and Elementary Volume Element with Isotropic Hardening}

Most of the authors use the elementary volume element containing one void, even widely used in the literature, but
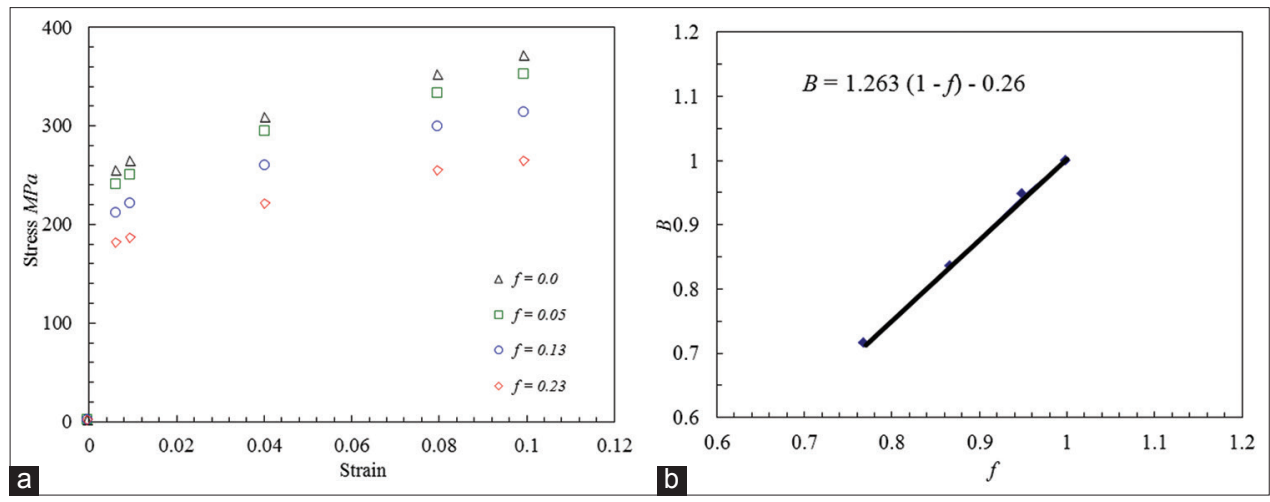

Figure 5: Verification of the calculation of the new parameter, (a) same strain for each porosity, and (b) relationship between new parameter and porosity, in different strains
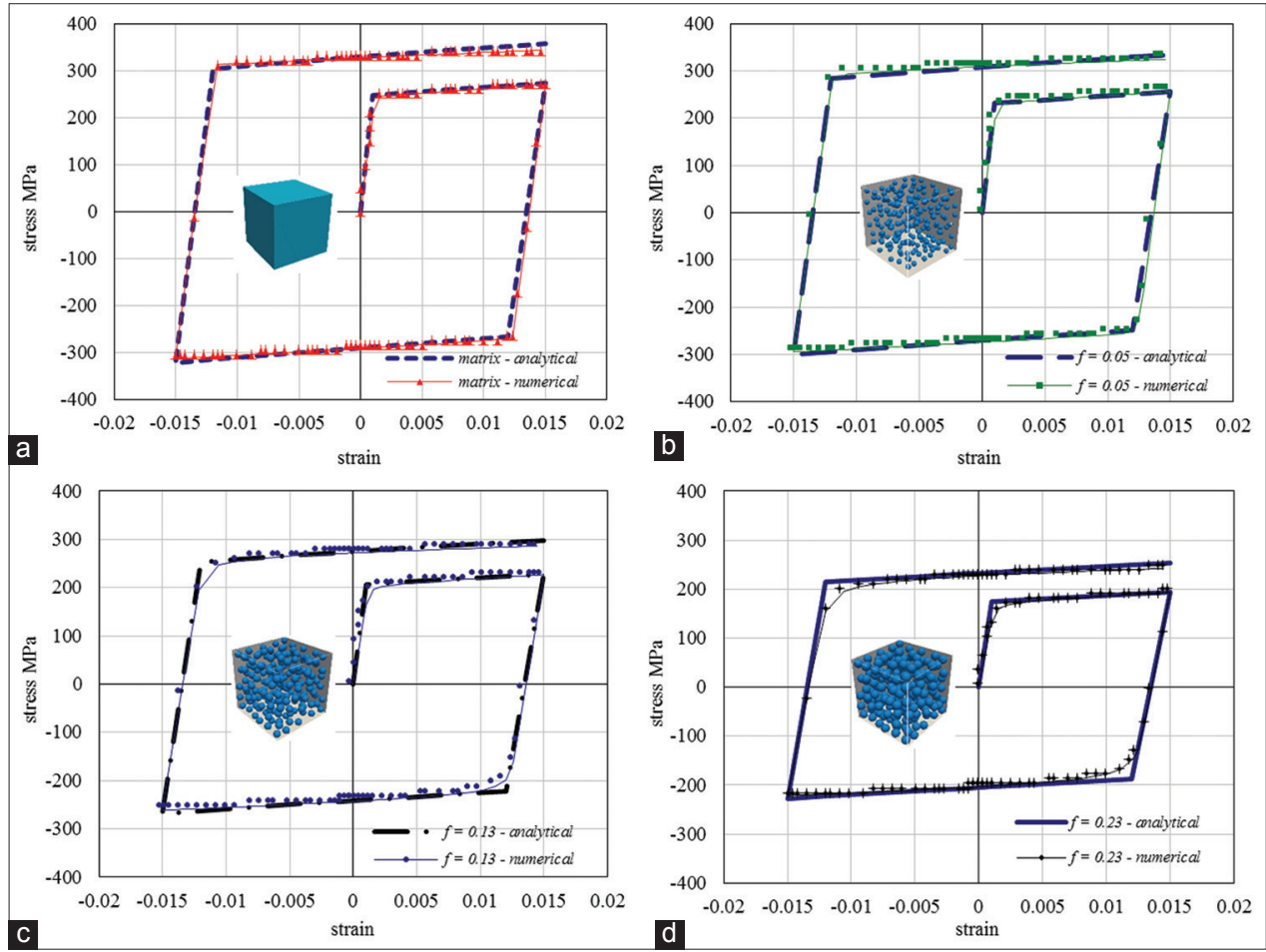

Figure 6: The comparison between the analytical and numerical results for different volume fractions; (a) analytical and numerical for matrix; analytical and numerical (b) $f=0.05$; (c) $f=0.13$; (d) $f=0.23$ 
always represent the minor bound of the real mechanical response in both cases tension or compression; the comparison between the results of elementary volume containing one void and the results of large RVE which contains 200 voids are explained, the difference between the two cases for three different volume fractions is indicated clearly; Figure 7a-c.

\section{Predicting the Linear Isotropic Plastic Hardening Modulus of Porous Materials}

The numerical finite element result which is corresponding 3D RVE microstructure is compared with analytical results using the same parameter $B$ which is, in this case, considered that there is plastic hardening. Two different volume fractions were tested for each case of the 10 different plastic hardening. Modulus $H$ was considered. Twenty numerical finite element tests (10) for each volume fraction compared with analytical model using Equation 16, and the same proposed parameter was used which it depends on the volume fraction. Moreover, plastic hardening modulus can be determined according to the following equation.

$$
\mathrm{H}_{\text {porous }}=\mathrm{H}_{\text {matrix }} \mathrm{B}
$$

and

$$
\left(\sigma_{\text {o }}\right)_{\text {porous }}=\left(\sigma_{\text {o }}\right)_{\text {matrix }} B
$$

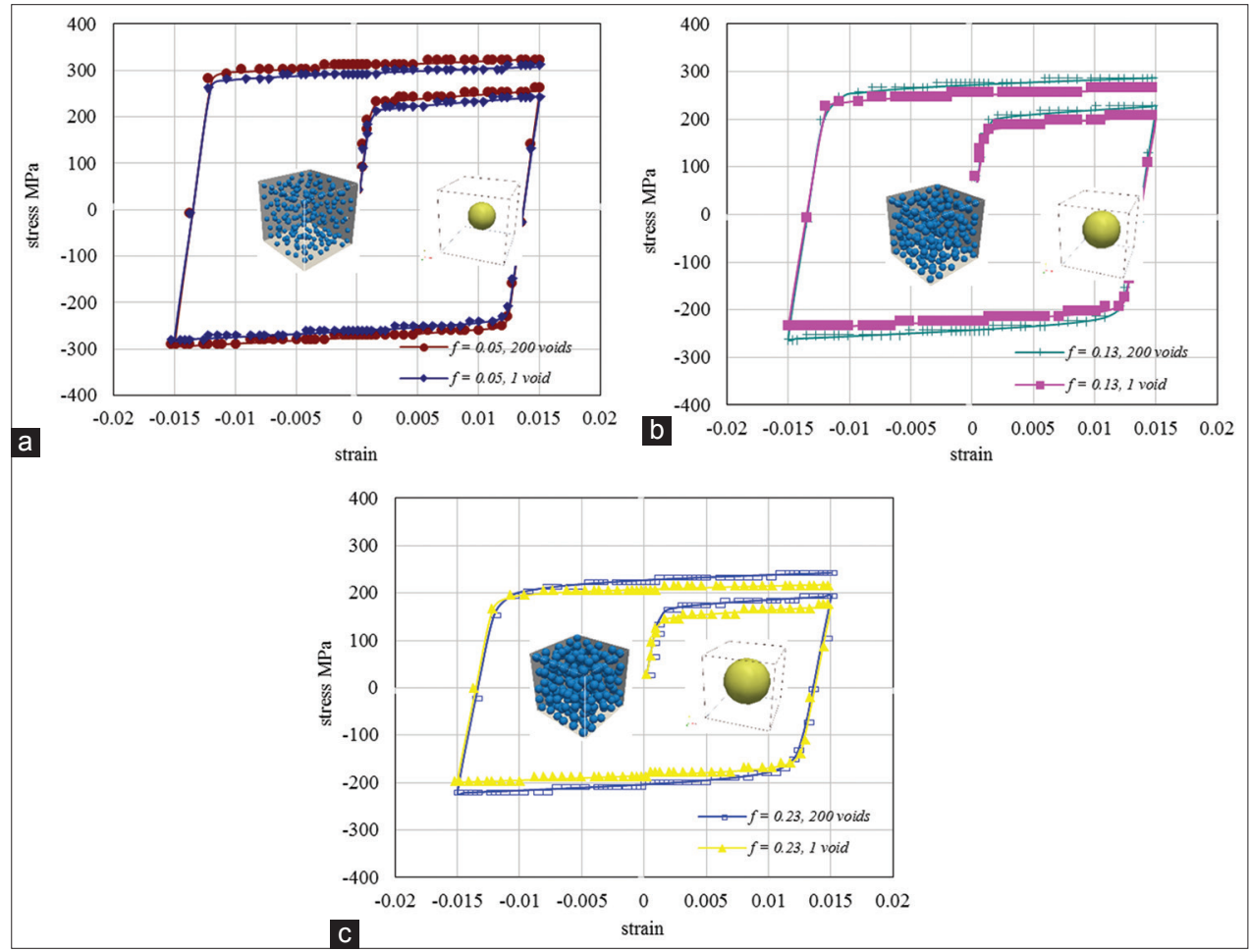

Figure 7: The differences between elementary volume element containing one void and RVE contains 200 voids for different volume fractions; (a) $f=0.05$, (b) $f=0.13$, and (c) $f=0.23$
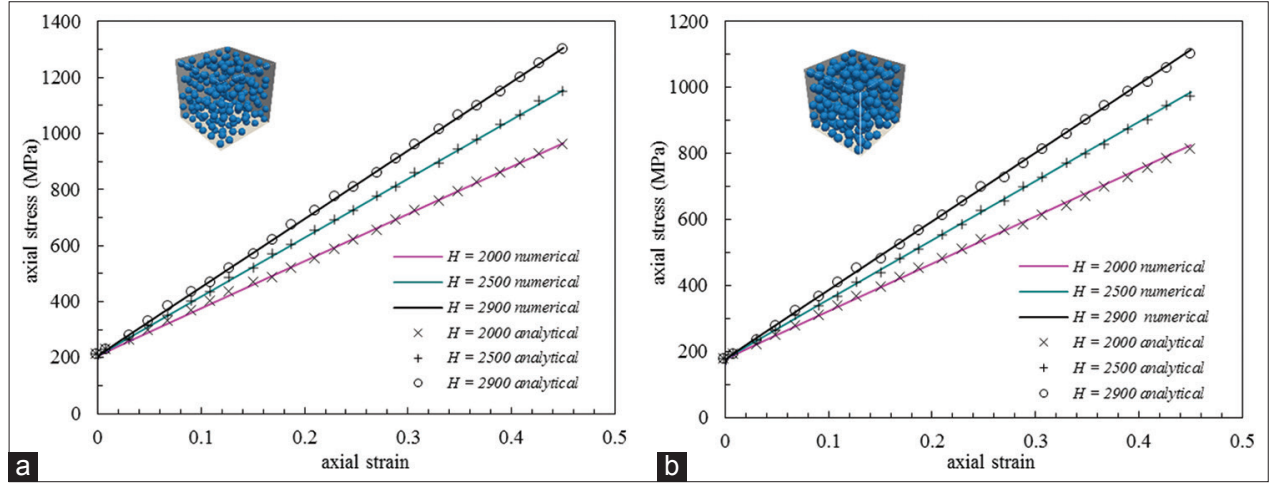

Figure 8: Examples of microstructures for different hardening modulus with volume fraction (a) $f=0.13$ and (b) $f=0.23$, and number of voids $n=200$, the lines represent numerical results and the other signs $(+, o, x)$ represent analytical results 

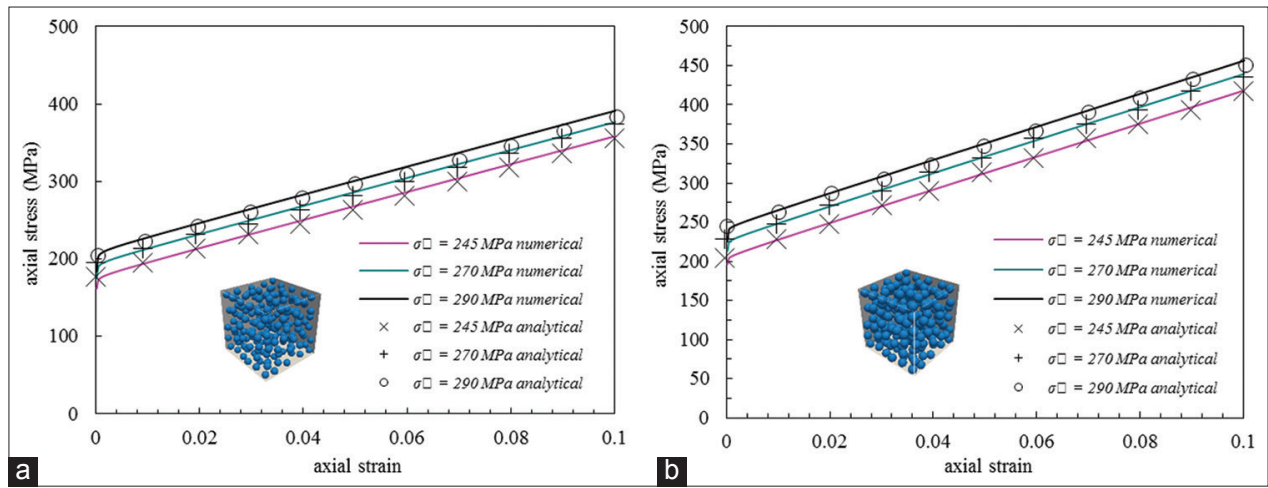

Figure 9: Examples of microstructures of different yield points with volume fraction (a) $f=0.13$ and (b) $f=0.23$, and number of voids $n=200$, the lines represent numerical results and other signs $(+, o, x)$ represent analytical results, and it considered isotropic hardening with the same modulus for all the tests

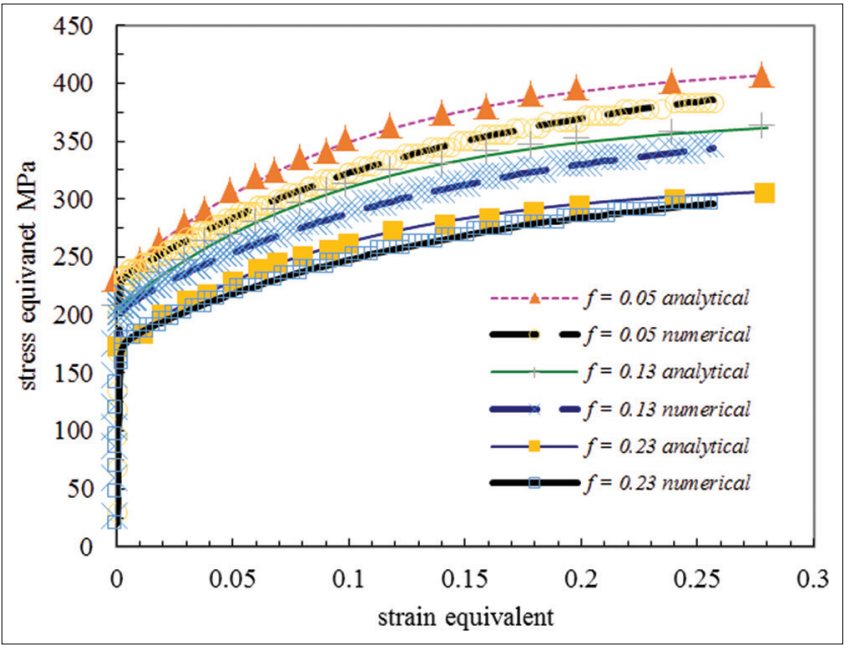

Figure 10: Comparison between analytical results and numerical finite element results for porous material with isotropic hardening in case of triaxial tension

Based on the linear isotropic hardening rule; for example, Chaboche, 1986, Simo and Hughes, 2006,

$$
\sigma_{\mathrm{Y}}=\left(\sigma_{\mathrm{o}}+H \varepsilon^{\mathrm{p}}\right) \mathrm{B}
$$

The above equation can be used for different volume fractions to evaluate the plastic hardening modulus for porous materials. The stress-strain curve for isotropic hardening porous materials can be determined by the equation below:

$$
\sigma_{\mathrm{Y}}=\left(\sigma_{\mathrm{o}}+H \varepsilon^{\mathrm{p}}\right) \mathrm{B}
$$

Where the slope of the curve is given by the constant plastic hardening modulus $H$, whereas $e^{p}$ denotes the accumulated or cumulative plastic strain, (Sorić et al., 2000, Besson and Guillemer-Neel, 2003, Steglich et al., 2005, Chaboche, 2008, Jin et al., 2008, Seifert and Schmidt, 2008, Seifert and Schmidt, 2009). Then, two types of volume fraction with 10 different plastic hardening modulus $H$, can justify the new proposed parameter $B$ during isotropic hardening. Examples of the results explained well in Figure 8, predicting plastic hardening modulus also one of the important steps so as to define the whole stress-strain curves for porous materials during isotropic hardening. Any change in the volume fraction leads to change the hardening parameter, i.e., the slope of the curves depends on the porosity of the materials directly.

\section{Predicting the Yield Stress for Porous Materials Considering Isotropic Hardening}

In this group of numerical tests, the author explain the determination of the yield stress and stress-strain curves of the porous materials, when there is the isotropic hardening, and author compared them with analytical results using Equation 16. Based on the numerical results, a good agreement is obtained between the numerical results and analytical results for several porous materials with different yield points, for each volume fraction 10 different yield stresses were tested, a total of 20 numerical tests showed that author can predict a yield stress of the porous material considering isotropic hardening analytically depending on the equation 16 examples are shown in Figure 9. Based on the numerical results, an important point can clarify that the yield stress significantly depends on the porosity of the materials. The increase of porosity leads to decrease in the effective yield stress, and vice versa.

\section{Predicting the Equivalent Stress-Strain Curves for Porous Materials, with Isotropic Hardening}

The equivalent stress $\Sigma_{e q}$, as explained in Equation 9, is described using the multi-component strain hardening model. The comparison between the analytical and numerical results is presented in Figure 10. The yield stress and strain hardening constants are fitted to the uniaxial stress-strain curves in a reference direction. To obtain only equivalent stress and the zero value of hydrostatic stress, the values of $\alpha, \beta$ should be as follows; $\alpha=1$ and $\beta=0$. 
Increasing the strain on the element leads to increase strain hardening up to $0.25 \%$ of the total equivalent strain and then became stable and continue because of the hardening saturation of the crystal grains. Based on the porosity of elements, the stress equivalent changes according to the ration of the volume fraction $f$. there is more agreement between the two results in the higher volume fraction $f=23$, while there is a little difference for lower porosity $f=5$.

\section{CONCLUSION}

In this study, a new analytical model is presented for predicting macroscopic behavior for porous materials, the matrix is considered elastoplastic with isotropic hardening and it fulfill the exponential law (Voce law) for isotropic hardening. A New Parameter $B$ added to the model. The proposed analytical model represents the extension of exponential law for isotropic hardening in, which can be used with the new added parameter for determination of macroscopic behavior of porous materials with isotropic hardening. The results of the new proposed analytical model compared with numerical results are obtained from calculating the finite element operation on a large RVE which contains 200 voids and also the results were compared with elementary volume element that contains only one centered void. Different volume fractions were tested numerically and analytically. Very good agreements between numerical and analytical results were found in each case of the large RVE that contains 200 voids, while the elementary volume element contains one void, given a minor bound. Based on the simulation, in this study, can use the proposed analytical model for a wide range of volume fractions between $f=0.05$ and $f=0.23$ to determine the macroscopic behavior for porous materials.

\section{REFERENCES}

Allain, S. and O. Bouaziz. 2008. Microstructure based modeling for the mechanical behavior of ferrite pearlite steels suitable to capture isotropic and kinematic hardening. Mater. Sci. Eng. A. 496(1-2): 329-336.

Balan, T. and O. Cazacu. 2013. Elastic plastic ductile damage model based on strain-rate plastic potential. Mech. Res. Commun. 54: 21-26.

Becker, R. and A. Needleman. 1986. Effect of yield surface curvature on necking and failure in porous plastic solids. J. Appl. Mech. 53(3): 491-499.

Berisha, B., P. Hora, A. Wahlen and L. Tong. 2010. A combined isotropic-kinematic hardening model for the simulation of warm forming and subsequent loading at room temperature. Int. J. Plast. 26(1): 126-140.

Besson, J. 2010. Continuum models of ductile fracture: A review. Int. J. Damage Mech. 19(1): 3-52.

Besson, J. and C. Guillemer-Neel. 2003. An extension of the green and gurson models to kinematic hardening. Mech. Mater.
35(1-2): 1-18.

Besson, J., D. Moinereau and D. Steglich. 2006. Local Approach to Fracture. Presses des MINES, France.

Buryachenko, V. A. 1996. The overall elastoplastic behavior of multiphase materials with isotropic components. Acta Mech. 119(1-4): 93-117.

Cao, J., W. Lee, H. S. Cheng, M. Seniw, H. P. Wang and K. Chung. 2009. Experimental and numerical investigation of combined isotropic-kinematic hardening behavior of sheet metals. Int. J. Plast. 25(5): 942-972.

Cardoso, R. P. and J. W. Yoon. 2009. Stress integration method for a nonlinear kinematic/isotropic hardening model and its characterization based on polycrystal plasticity. Int. J. Plast. 25(9): 1684-1710.

Carollo, V., M. Paggi and A. Rossani. 2016. A two parameter elastoplastic formulation for hardening pressure-dependent materials. Mech. Res. Commun. 77: 1-4.

Chaaba, A. 2013. Reliability assessment by analytical calculation of the plastic collapse load of thin pressure vessels with strain hardening and large deformation. Thin Walled Struct. 62: 46-52.

Chaboche, J. and O. Jung. 1997. Application of a kinematic hardening viscoplasticity model with thresholds to the residual stress relaxation. Int. J. Plast. 13(10): 785-807.

Chaboche, J. L. 1986. Time-independent constitutive theories for cyclic plasticity. Int. J. Plast. 2(2): 149-188.

Chaboche, J. L. 2008. A review of some plasticity and viscoplasticity constitutive theories. Int. J. Plast. 24(10): 1642-1693.

Chaboche, J. L., Kanouté, P and F. Azzouz. 2012. Cyclic inelastic constitutive equations and their impact on the fatigue life predictions. Int. J. Plast. 35: 44-66.

Chawla, N. and X. Deng. 2005. Microstructure and mechanical behavior of porous sintered steels. Mater. Sci. Eng. A. 390(1-2): 98-112.

Chow, C. and X. Yang. 2003. Prediction of forming limit diagram with mixed anisotropic kinematic isotropic hardening plastic constitutive model based on stress criteria. J. Mater. Process. Technol. 133(3): 304-310.

De Angelis, F. 2012. A comparative analysis of linear and nonlinear kinematic hardening rules in computational elastoplasticity. Technische Mech., 32(2-5): 164-173.

Doghri, I. and A. Ouaar. 2003. Homogenization of two-phase elastoplastic composite materials and structures: Study of tangent operators, cyclic plasticity and numerical algorithms. Int. J. Solids Struct. 40(7): 1681-1712.

Doghri, I. and C. Friebel. 2005. Effective elasto-plastic properties of inclusion-reinforced composites. Study of shape, orientation and cyclic response. Mech. Mater. 37(1): 45-68.

Dung, N. L. 1992. Three-dimensional void growth in plastic materials. Mech. Res. Commun. 19(3): 227-235.

Fritzen, F., S. Forest, T. Böhlke, D. Kondo and T. Kanit. 2012. Computational homogenization of elasto-plastic porous metals. Int. J. Plast. 29: 102-119.

Gurson, A. L. 1977. Continuum theory of ductile rupture by void nucleation and growth: Part I yield criteria and flow rules for porous ductile media. J. Eng. Mater. Technol. 99(1): 2-15.

Hashemi, E. and B. Farshi. 2011. Cyclic loading of beams based on kinematic hardening models: A finite element approach. Int. J. Model. Optim. 1(3): 210.

Jiang, T., J. F. Shao and W. Xu. 2011. A micromechanical analysis of elastoplastic behavior of porous materials. Mech. Res. Commun. 38(6): 437-442. 
Jin, C., J. Niu, S. He and C. Fu. 2008. Modeling thermal cycling induced micro-damage in aluminum welds: An extension of Gurson void nucleation model. Comput. Mater. Sci. 43(4): 1165-1171.

Khan, A. S. and K. M. Jackson. 1999. On the evolution of isotropic and kinematic hardening with finite plastic deformation Part I: compression/tension loading of OFHC copper cylinders. Int. J. Plast. 15(12): 1265-1275.

Khdir, Y. K., T. Kanit, F. Zaïri and M. Naït-Abdelaziz. 2014. Computational homogenization of plastic porous media with two populations of voids. Mater. Sci. Eng. A. 597: 324-330.

Khdir, Y. K., T. Kanit, F. Zaïri and M. Naït-Abdelaziz. 2015. A computational homogenization of random porous media: Effect of void shape and void content on the overall yield surface. Eur. J. Mech. A Solids. 49: 137-145.

Khdir, Y., T. Kanit, F. Zaïri and M. Nait-Abdelaziz. 2013. Computational homogenization of elastic plastic composites. Int. J. Solids Struct. 50(18): 2829-2835.

Khoei, A. and A. Azami. 2005. A single cone-cap plasticity with an isotropic hardening rule for powder materials. Int. J. Mech. Sci. 47(1): 94-109.

Kossa, A. and L. Szabó. 2009. Exact integration of the von Mises elastoplasticity model with combined linear isotropic-kinematic hardening. Int. J. Plast. 25(6): 1083-1106.

Kuna, M. and D. Sun. 1996. Three-dimensional cell model analyses of void growth in ductile materials. Int. J. Fract. 81(3): 235-258.

Lademo, O. G., O. S. Hopperstad and M. Langseth. 1999. An evaluation of yield criteria and flow rules for aluminium alloys. Int. J. Plast. 15(2): 191-208.

Leblond, J. B., G. Perrin and J. Devaux. 1995. An improved Gursontype model for hardenable ductile metals. Eur. J. Mech. A Solids. 14(4): 499-527.

Leu, S. Y. and R. S. Li. 2012. Exact solutions of sequential limit analysis of pressurized cylinders with combined hardening based on a generalized hölder inequality: Formulation and validation. Int. J. Mech. Sci. 64(1): 47-53.

Mahmoudi, A., S. Pezeshki-Najafabadi and H. Badnava. 2011. Parameter determination of chaboche kinematic hardening model using a multi objective genetic algorithm. Comput. Mater. Sci. 50(3): 1114-1122.

Mear, M. and J. Hutchinson. 1985. Influence of yield surface curvature on flow localization in dilatant plasticity. Mech. Mater. 4(3-4): 395-407.

Mosler, J. 2010. Variationally consistent modeling of finite strain plasticity theory with non-linear kinematic hardening. Comput. Methods Appl. Mech. Eng. 199(45-48): 2753-2764.

Rezaiee-Pajand, M. and S. Sinaie. 2009. On the calibration of the chaboche hardening model and a modified hardening rule for uniaxial ratcheting prediction. Int. J. Solids Struct. 46(16): 3009-3017.

Ristinmaa, M. 1995. Cyclic plasticity model using one yield surface only. Int. J. Plast. 11(2): 163-181.

Ristinmaa, M. 1997. Void growth in cyclic loaded porous plastic solid. Mech. Mater. 26(4): 227-245.

Rousselier, G., F. Barlat and J. W. Yoon. 2010. A novel approach for anisotropic hardening modeling. Part II: Anisotropic hardening in proportional and non-proportional loadings, application to initially isotropic material. Int. J. Plast. 26(7): 1029-1049.

Samrout, H., R. El Abdi and J. Chaboche. 1997. Model for 28CrMoV5-8 steel undergoing thermomechanical cyclic loadings. Int. J. Solids Struct. 34(35-36): 4547-4556.

Seifert, T. and I. Schmidt. 2008. Line-search methods in general return mapping algorithms with application to porous plasticity. Int. J. Numer. Methods Eng. 73(10): 1468-1495.

Seifert, T. and I. Schmidt. 2009. Plastic yielding in cyclically loaded porous materials. Int. J. Plast. 25(12): 2435-2453.

Siad, L., W. K. Liu and A. Benabbes. 2009. Explicit numerical study of softening in porous ductile solids. Mech. Res. Commun. 36(2): 236-245.

Simo, J. C. and T. J. Hughes. 2006. Computational Inelasticity. Springer Science and Business Media, Berlin.

Sorić, J., Z. Tonković and W. Krätzig. 2000. A new formulation of numerical algorithms for modelling of elastoplastic cyclic response of shell-like structures. Comput. Struct. 78(1-3): 161-168.

Steglich, D., A. Pirondi, N. Bonora and W. Brocks. 2005. Micromechanical modelling of cyclic plasticity incorporating damage. Int. J. Solids Struct. 42(2): 337-351.

Taherizadeh, A., A. Ghaei, D. E. Green and W. J. Altenhof. 2009. Finite element simulation of springback for a channel draw process with drawbead using different hardening models. Int. J. Mech. Sci. 51(4): 314-325.

Tvergaard, V. 1982. On localization in ductile materials containing spherical voids. Int. J. Fract. 18(4): 237-252.

Tvergaard, V. and A. Needleman. 1984. Analysis of the cup-cone fracture in a round tensile bar. Acta Metall. 32(1): 157-169.

Verleene, A., L. Dubar, A. Dubois, M. Dubar and J. Oudin. 2002. Hardening behaviour law versus rigid perfectly plastic law: Application to a cold forging tool steel. Int. J. Plast. 18(8): 997-1011.

Verma, R. K., T. Kuwabara, K. Chung and A. Haldar. 2011. Experimental evaluation and constitutive modeling of nonproportional deformation for asymmetric steels. Int. J. Plast. 27(1): 82-101.

Voyiadjis, G. Z. and C. R. Song. 2002. Multi-scale non-local approach for geomaterials. Mech. Res. Commun. 29(2-3): 121-129.

Zhang, Z., Z. Zhuang, Y. Gao, Z. Liu and J. Nie. 2011. Cyclic plastic behavior analysis based on the micromorphic mixed hardening plasticity model. Comput. Mater. Sci. 50(3): 1136-1144.

Zhao, K. and J. Lee. 2001. Material properties of aluminum alloy for accurate draw-bend simulation. J. Eng. Mater. Technol. 123(3): 287-292. 